\title{
Sub-degree CMB anisotropy from space
}

\section{In-flight calibration}

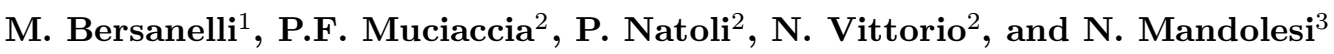 \\ 1 Istituto di Fisica Cosmica, CNR, Milano, Italy \\ 2 Dipartimento di Fisica, Universita' di Tor Vergata, Roma, Italy \\ 3 Istituto TESRE, CNR, Bologna, Italy
}

Received March 7; accepted June 7, 1996

\begin{abstract}
In the context of the COBRAS/SAMBA mission study $^{1}$, we discuss in-flight calibration of extended sky maps of the microwave sky using celestial sources. We simulate the observations in order to assess the accuracy obtainable for absolute and relative calibration of the Low Frequency Instrument (LFI), operating in the $30-130 \mathrm{GHz}$ range. Accurate calibration can be achieved using the CMB dipole signal, $\Delta T_{\mathrm{D}}$. With conservative assumptions on the effect of Galactic contamination, we find that the $\mathrm{CMB}$ dipole will provide absolute calibration accuracy $\sim 0.7 \%$ (limited by the COBE-DMR uncertainty on $\Delta T_{\mathrm{D}}$ ) on time-scales of about 10 days at all frequencies and for the entire mission lifetime. Long-term calibration with accuracy $<0.2 \%$ can be obtained using the spacecraft orbital velocity. Additional, independent calibration will be provided by the observation of external planets. We also describe the capability of the proposed scanning technique to detect and remove long-term instrumental drifts, and show that these effects, if present, can be controlled and removed with an overall negligible impact on the data uncertainty.
\end{abstract}

Key words: cosmic microwave background - radio continuum: general - space vehicles

\section{Introduction}

One of the most promising objectives of observational cosmology achievable in the next decade is an extensive ( $>90 \%$ of the sky), accurate $\left(\Delta T / T \approx 10^{-6}\right)$, detailed (angular resolution $<30^{\prime}$ ) imaging of the Cosmic Microwave Background (CMB) anisotropies. A precise reconstruction of the primordial fluctuation spectrum up to multipoles of order $\ell \sim 10^{3}$ (corresponding to angular

Send offprint requests to: M. Bersanelli scales $\sim 0.1^{\circ}$ ) provides a unique tool to gain new insights on fundamental issues of physics and cosmology. Following the COBE-DMR detection of anisotropy $\left(\Delta T / T \approx 10^{-5}\right)$ at large $\left(>7^{\circ}\right)$ angular scales (Smoot et al. 1992), an increasing number of experiments have been performed with ground-based and balloon-borne instruments at degree angular scales (e.g., Cheng et al. 1994; De Bernardis et al. 1994; Clapp et al. 1994; Gundersen et al. 1995; Ruhl et al. 1995; Netterfield et al. 1995, and references therein). A second-generation space mission, however, is required to fully image the CMB anisotropies with sub-degree angular resolution (e.g. Danese et al. 1995). It has been shown (e.g. Jungman et al. 1996) that accurate full-sky maps with resolution $<30^{\prime}$ yield precise determination of all key cosmological parameters, such as the total and baryon density parameters $\Omega_{0}$ and $\Omega_{B}$, the Hubble constant $H_{0}$, the cosmological constant $\Lambda$. These parameters and a statistical analysis on the detected anisotropies will allow to trace structure formation mechanisms and the thermal history of the universe, while producing effective tests of inflation. A number of space projects are under study in Europe and in the U.S.A. to address this outstanding scientific goal (Mandolesi et al. 1995; Bouchet et al. 1995; Bersanelli et al. 1996; Janssen \& Lawrence 1995; Wright et al. 1996).

An important aspect to consider in the design of the space-borne instrument and observation technique is the accuracy and reliability of the method for calibrating the detected sky signals. In this paper we analyze the calibration issue based on the COBRAS/SAMBA ${ }^{1}$ Low Frequency Instrument (LFI) concept. details of our analysis will depend on this the general results will be of The LFI concept and the COBRAS/SAMBA mission have been described elsewhere (Bersanelli et al. 1995, 1996; Mandolesi et al. 1995; Muciaccia et al. 1996), and here we only outline the main features. The LFI is an

1 COBRAS/SAMBA is an European Space Agency candidate mission for the M 3 program, devoted to high-resolution imaging of the CMB anisotropy. 
array of 28 corrugated horns, each feeding two independent

receivers, at the focal plane of an off-axis optical system. The array works in four frequency bands (centered at 31.5, $53,90$, and $125 \mathrm{GHz})$ with passively cooled $(\sim 100 \mathrm{~K})$ differential radiometers based on state-of-the-art transistor amplifiers. Different channels have different angular resolution: $30^{\prime}, 18^{\prime}, 12^{\prime}$ and $12^{\prime}$ FWHM for the 31.5, 53, 90 and $125 \mathrm{GHz}$ channels, respectively. The spacecraft is spin-stabilized with a spin rate of $1 \mathrm{rpm}$, and the telescope field of view is offset by $70^{\circ}$ with respect to the spin axis. It will operate from a Lissajous orbit around the L2 point of the Sun-Earth system, pointed in anti-Sun direction during normal operation. In the baseline scan strategy the spin axis will be re-oriented by $5^{\prime}$ every 2 hours, to compensate for the Earth revolution and keep the anti-sun position $^{2}$. Therefore, each detector observes the same sky circle, approximately $140^{\circ}$ wide, for two hours before each $5^{\prime}$ step.

The output of the $n$-th radiometer channel $(1 \leq n \leq$ 56 ) will be a time-dependent voltage $V_{n}(t)$, expressed in terms of telemetry counts. The purpose of calibration is to convert the signal strength from raw telemetry data into physical units. The calibration is performed in terms of antenna temperature, proportional to the received power per unit bandwidth. If a linear receiver (the system is designed to be linear in its operating range) observes during a scan two sources of known antenna temperatures $T_{1}$ and $T_{2}$ at times $t_{1}$ and $t_{2}$, the calibration constant, or gain, is determined by:

$G_{n}=\frac{T_{1}-T_{2}}{V_{n}\left(t_{1}\right)-V_{n}\left(t_{2}\right)} \equiv \frac{\Delta T}{\Delta V_{n}}$.

In principle, each value $G_{n}$ is constant in time; in practice, temperature variations or intrinsic instrumental effects may produce drifts or time fluctuations. Thus one wishes to measure the calibration constants $G_{n}$ as accurately as possible and as frequently as possible during the mission, and/or monitor the stability of $G_{n}$ with time. Following Bennett et al. (1992), we use the term absolute calibration to refer to a measurement of the value of $G_{n}$, and relative calibration to refer to a measurement of its time-stability.

The calibrating signal can be provided by stable solidstate noise sources, a strategy adopted by the COBEDMR experiment (Smoot et al. 1990; Bennett et al. 1992). However, for an experiment like COBRAS/SAMBA the implementation of a suitable active calibration system is likely to require some compromise in instrument performance (either additional insertion loss in the front-end low-noise amplifiers for internal sources, or some level of distortion in the optical quality of the telescope for external sources). In any case, it is important to evaluate

2 Pointing maneuvers up to $\pm 15^{\circ}$ around the anti-sun position are allowed, and an optimized mission plan for best sky coverage is currently under study (Mandolesi et al. 1996). the accuracy achievable using celestial sources which will be observed during the survey. In this work we limit the analysis to calibration with celestial sources. In Sect. 2 we study the accuracy which can be achieved in the determination of $G_{n}$ using the CMB dipole, the spacecraft orbital velocity and the signal from external planets.

The rest of this work deals with our ability to control long-term variation of the instrumental response. The LFI receivers (Bersanelli et al. 1995) measure signals $V_{n}$ proportional to the antenna temperature difference between the sky at a given frequency, $T_{\text {sky }}$, and a stable internal load associated with each receiver at a comparable effective temperature, $T_{\mathrm{eff}, n}$ :

$V_{n}=\frac{1}{G_{n}}\left(T_{\mathrm{sky}}-T_{\mathrm{eff}, n}\right)$.

Thus, both changes in $G_{n}$ and drifts in $T_{\text {eff }, n}$ can perturb the measured signal $V_{n}$. The two effects, however, can be decoupled. Since in a time interval $\lesssim 1$ min we can assume that the instrument response is stable, the signal difference between two sky regions $T_{\text {sky }}^{(1)}$ and $T_{\text {sky }}^{(2)}$ observed in the same scan circle can be written as

$\Delta V_{n}^{(2,1)}=\frac{1}{G_{n}}\left(T_{\text {sky }}^{(2)}-T_{\text {sky }}^{(1)}\right)$,

which is independent of $T_{\text {eff }, n}$. Thus one can recognize the effect of a change in the calibration constant as a timevariation of the quantity $\Delta V_{n}^{(2,1)}$, i.e., a change in the signal differences corresponding to two (or more) fixed regions of the sky. On the other hand, a change in the observed signal $V_{n}$ which leaves $\Delta V_{n}^{(2,1)}$ unchanged is the signature of a thermal variation of the effective load temperature $T_{\text {eff, } n}$. In Sects. 3 and 4 , we discuss the effects of long-term gain drifts (relative calibration) and thermal baseline changes, respectively.

\section{Absolute calibration}

The purpose of an absolute calibration is the determination of the values $G$ (hereafter we refer to a given channel and drop the subscript $n$ ) according to Eq. (1) through the measurement of (at least) two signals corresponding to well known intrinsic antenna temperatures $T_{1}$ and $T_{2}$. If their difference $\Delta T$ is a priori known with an uncertainty $\sigma_{\Delta T}$, from Eq. (1) we find the uncertainty on $G$ to be

$$
\begin{aligned}
\sigma_{G} & =\left[\left(\frac{1}{\Delta V} \sigma_{\Delta T}\right)^{2}+\left(-\frac{\Delta T}{\Delta V^{2}} \sigma_{\Delta V}\right)^{2}\right]^{1 / 2} \\
& =\left[\left(\frac{G}{\Delta T} \sigma_{\Delta T}\right)^{2}+\left(\frac{G^{2}}{\Delta T} \sigma_{\Delta V}\right)^{2}\right]^{1 / 2}
\end{aligned}
$$

The measured voltage difference has a spread

$\sigma_{\Delta V}=\sqrt{2} \frac{\delta T_{\mathrm{rms}}(\tau)}{G}$ 
Here $\delta T_{\text {rms }}(\tau)$ is the rms noise associated with the measurement of $T_{1}$ or $T_{2}$ in an integration time $\tau$ :

$\delta T_{\mathrm{rms}} \simeq K \frac{T_{\mathrm{sys}}}{\sqrt{\Delta \nu \tau}}$,

where $K$ is a constant of order unity depending on the type of receiver (for the differential baseline LFI receivers $K=$ $\sqrt{2}$ ), $\Delta \nu$ is the bandwidth, and $T_{\text {sys }}$ is the system noise temperature. Therefore, to first order, the uncertainty in the absolute calibration is given by:

$$
\left(\frac{\sigma_{G}}{G}\right)_{\mathrm{abs}} \simeq \frac{\sqrt{\sigma_{\Delta T}^{2}+2 \delta T_{\mathrm{rms}}^{2}}}{\Delta T}
$$

or, according to Eq. (6),

$$
\left(\frac{\sigma_{G}}{G}\right)_{\mathrm{abs}} \simeq \frac{1}{\Delta T} \sqrt{\sigma_{\Delta T}^{2}+2 K^{2} \frac{T_{\mathrm{sys}}^{2}}{\Delta \nu \tau}} .
$$

From Eq. (7) it appears that a large calibrating signal can produce higher accuracies for a given integration time and sensitivity. However, the useful amplitude of the signal is limited by saturation effects occurring in the amplification chains of the radiometers.

\subsection{Absolute calibration using the $C M B$ dipole}

Because of our peculiar motion relative to the comoving frame, the CMB Planckian radiation field of temperature is observed to have a temperature

$T(\hat{\gamma})=T_{\mathrm{CMB}}+\Delta T_{\mathrm{D}} \times(\hat{\gamma} \cdot \hat{n})$.

Here $T_{\mathrm{CMB}}=2.726 \pm 0.010 \mathrm{~K}$ is the CMB temperature (Mather et al. 1994) while $\hat{\gamma}$ and $\hat{n}$ are the observing line of sight and the direction of our motion relative to the comoving frame, respectively. The amplitude $\left(\Delta T_{\mathrm{D}}=3.358 \pm 0.023 \mathrm{mK}\right)$ and direction $([l, b]=$ $\left.\left[264.31^{\circ} \pm 0.17^{\circ}, 48.05^{\circ} \pm 0.09^{\circ}\right]\right)$ of the $\mathrm{CMB}$ dipole have been accurately measured by the COBE-DMR instruments (Kogut et al. 1993; Lineweaver et al. 1996). The dipole emission will show up in the LFI data as a continuous modulation of the signals $V(t)$ with accurately known amplitude. The CMB dipole is thus a particularly attractive source as it can be used for calibration without reducing the efficiency of the observations.

For a first-order evaluation of the achievable accuracy, we have calculated the antenna temperature difference, $\Delta T_{\mathrm{D}}=T\left(\hat{\gamma}_{\max }\right)-T\left(\hat{\gamma}_{\min }\right)$, between the maximum and the minimum of the dipole modulation for each spin rotation during the first year of the mission (2003), as seen by each channel at the four LFI frequencies. We then estimated $\sigma_{G}$ from Eq. (7) assuming $\Delta T=\Delta T_{\mathrm{D}}$ in a portion of $\pm 10^{\circ}$ around $\hat{\gamma}_{\max }$ and $\hat{\gamma}_{\min }$, respectively. This rough calculation suggests that the CMB dipole allows for absolute calibration accuracies better than $3 \%$ for all LFI channels, at a rate of about one day. This estimate, however, besides the simplifying assumption in the fit, neglects the presence of the Galactic emission which is mixed to the dipole signal. The Galactic contribution is known with relatively poor accuracy in the frequency range of interest $(30-140 \mathrm{GHz})$, making the regions with strong Galactic signal useless for accurate absolute calibration.

We performed a more refined study by simulating the microwave sky (CMB dipole and Galaxy) as observed at each LFI frequency, and by reproducing the actual $70^{\circ}$ offset, $1 \mathrm{rpm}$ scan pattern of the LFI array. We model the Galactic contribution including the synchrotron, freefree and dust components. The synchrotron emission at the LFI frequencies is obtained by properly rescaling the $408 \mathrm{MHz}$ full-sky survey of Haslam et al. (1982):

$\Delta T_{\text {syn }}(\nu)=T(408 \mathrm{MHz}) \frac{\left(\mathrm{e}^{x}-1\right)^{2}}{x^{2} \mathrm{e}^{x}}\left(\frac{\nu}{408 \mathrm{MHz}}\right)^{\alpha_{\mathrm{syn}}}$,

where $x \equiv h \nu / k T_{\mathrm{CMB}}$, and the spectral index $\alpha_{\text {syn }}$ is assumed to have a constant value over the sky. Although pixel-to-pixel variations of the spectral index are expected, as suggested by a comparison of the Haslam $408 \mathrm{MHz}$ and Reich and Reich 1.4 GHz maps (Lawson et al. 1987), our results are not affected significantly by this effect. We consider the two values $\alpha_{\mathrm{syn}}=-2.9$, the upper limit recently derived by Kogut et al. (1996) from the COBE data, and $\alpha_{\text {syn }}=-2.75$, a more "conservative" value commonly used in the literature. the results The angular resolution of the synchrotron emission has been artificially increased by interpolating the $408 \mathrm{MHz}$ map on pixels of sizes equal to the FWHM of the antenna beams. To account for free-free emission we considered both a diffuse component [with a $\operatorname{cosec}(b)$ dependence] and a compilation of $\sim 800 \mathrm{HII}$ regions near the Galactic plane (Witebsky 1988). The HII regions have been first convoluted with the angular response of the antenna beam, approximated with a Gaussian of dispersion $\sigma_{B}=\mathrm{FWHM} /(2 \sqrt{2 \ln 2})$. We extrapolated free-free emission to the observing frequencies by assuming a spectral index $\alpha_{\mathrm{ff}}=-2.1$. The dust emission has been modeled by extrapolating the IRAS $100 \mu \mathrm{m}$ map with a spectral index $\alpha_{\mathrm{d}}=1.5$.

Accurate calibration needs to take into account the presence of the CMB fluctuations themselves. High order multipoles should not affect significantly the dipole calibration, since their global contribution to the dipole reconstruction will tend to average out. On the other hand, ignoring the quadrupole and other low- $\ell$ CMB components would result in a significant error in the calibration (of order of $1 \%$ ), and the a-priori accuracy in the value of these terms as determined by COBE is relatively poor. However, it will be possible to construct an iterative algorithm yielding the CMB sky fluctuations and the calibration by successive approximations. In these simulations the presence of the CMB cosmological quadrupole and higher order multipoles was not included.

The following algorithm was constructed to simulate the observations. We sample the circle scanned by the 
beam with $N$ independent elements (or "pixels"), where $N=720$ at $31.5 \mathrm{GHz}, N=1200$ at $53 \mathrm{GHz}$ and $N=1800$ at 90 and $125 \mathrm{GHz}$. Thus, the simulated output of a given scan is a set of $N$ voltages $V_{1}, V_{2}, \ldots V_{i}, \ldots V_{N}$ corresponding to the expected sky temperature (dipole plus Galaxy) in each direction, to which we add a Gaussian noise $\sigma_{i}$ characteristic of each radiometer at the proper integration time:

$V_{i}=\frac{1}{G}\left(T_{\mathrm{D}, i}+T_{\mathrm{Gal}, i}\right)+\sigma_{i}$.

For each pixel in a given circle, we then calculate the simulated signal difference

$\Delta V_{i}=V(\hat{\gamma})-V(-\hat{\gamma})$

between opposite pixels on the circle. To minimize Galactic contamination, the procedure eliminates all the pixels for which the expected Galactic temperature difference, $\Delta T_{\mathrm{Gal}, i}$, is a significant fraction, $\rho$, of the $\mathrm{CMB}$ dipole temperature difference, $\Delta T_{\mathrm{D}, i}$. The value of this threshold $\rho \equiv \Delta T_{\mathrm{Gal}, i} / \Delta T_{\mathrm{D}, i}$ was left as an adjustable parameter. We assume here that $G$ is stable over the time scale of the absolute calibration (in the next sections we will discuss correction of long-term gain drifts). Thus every 120 minutes we have a set of $N / 2$ differences, $\Delta V_{i}$, with a dispersion $\sigma_{i}=\sqrt{2} \delta T_{\text {rms }} / \sqrt{120}$, where $\delta T_{\text {rms }}$ is the instrumental noise per pixel (Eq. 6). We perform the absolute calibration by minimizing

$\chi^{2}=\sum_{i=1}^{N / 2} \frac{\left(\Delta V_{i}\left(t_{k}\right)-G^{-1} \Delta T_{\mathrm{D}, i}\right)^{2}}{\sigma_{i}^{2}}$

where $\Delta T_{\mathrm{D}, i}$ is the a-priori-known value of the dipole temperature difference corresponding to the observed directions.

It is clear that we can neither increase nor decrease too much the threshold $\rho$. In fact, in the first case the number of surviving pixel pairs tends to zero; in the second case the number of pixel pairs contaminated by the Galactic emission will be large, and the minimization of the $\chi^{2}$ will yield answers affected by systematic errors. It turns out that in order to achieve accurate calibration one needs $\rho \simeq$ $0.003-0.02$, depending on the frequency. Our results are shown in Fig. 1. The frequency of the calibration and the choice of the threshold $\rho$ have been set at each frequency aiming at an upper limit to the uncertainty $<0.7 \%$, which corresponds to the intrinsic limit of the accuracy of $\Delta T_{\mathrm{D}}$ as measured by COBE-DMR. At the highest frequencies (90 and $125 \mathrm{GHz}$ ) the Galaxy is relatively unimportant. The calibration constant is recovered within $\sim 0.3 \%$ every 10 days for most of the mission lifetime, except in two periods (approximately around days 70 and 250), which correspond to those circles where the differential dipole signal, as observed by the LFI detectors, has a minimum. This confirms the comment to Eq. (7) above.
At $31.5 \mathrm{GHz}$, in order to avoid contamination from the Galaxy a severe threshold $\rho=0.003$ is needed. For this reason one can derive $G$ at the same accuracy level only once every $\sim 20$ days. The fit is achieved again by minimizing Eq. (13), where the sum is now extended over the "clean" pixels of 264 adjacent circles.

A comparison between the results for $\alpha_{\text {syn }}=-2.9$ and $\alpha_{\text {syn }}=-2.75$ indicates that the dependence on the assumed Galactic model is weak. Note that we have not attempted any subtraction of the Galactic components. New accurate observations of the Galactic foregrounds, expected as part of a mission like COBRAS/SAMBA, will allow to construct an iterative subtraction procedure which will improve the calibration accuracy by further reducing systematic errors.

Performing the fits over more extended time periods can largely reduce the statistical error. For example, at $31.5 \mathrm{GHz}$, with a threshold $\rho=0.01$ the statistical error is below $0.1 \%$ when considering 1 year of observations (i.e. just one calibration per year). However, the ultimate limit to the overall accuracy in the absolute calibration is set by the uncertainty of a-priori knowledge of the CMB dipole amplitude and direction. The four-year COBE-DMR data yield a best fit dipole amplitude with a $\sim 0.7 \%$ accuracy (Lineweaver et al. 1996). Position uncertainties will be dominated by the COBE-DMR $\sim 0.15^{\circ}$ error in the absolute $(\ell, b)$ dipole direction, which propagates to $<0.1 \%$ error. Due to the slow angular gradient of the dipole, the COBRAS/SAMBA pointing uncertainties (2.5' in 30 minutes) will result in a negligible contribution (less than $0.03 \%$ ) to the total error.

We can conclude that the CMB dipole will allow frequent ( $\sim 10$ days) absolute calibration reaching the limit set by the intrinsic uncertainty on the calibrating signal. This uncertainty induces a $\lesssim 1 \mu \mathrm{K}$ uncertainty in the amplitude of the small scale CMB anisotropy, which is adequate to the mission goal.

\subsection{Dipole modulation due to the spacecraft velocity}

To push the long-term ( $~ \& 1$ year) calibration accuracy beyond the COBE-DMR uncertainty in the CMB dipole amplitude, we can use the Doppler effect due to the satellite orbital velocity. This effect was also used by COBEDMR for absolute calibration (Kogut et al. 1996). In the case of COBRAS/SAMBA, after correction for the small $(<0.2 \mathrm{~km} / \mathrm{s})$ component of the satellite motion in the L2 rest frame, the seasonal velocity component around the Sun $\left(\simeq 30 \mathrm{~km} / \mathrm{s}\right.$. on average, or $\left.\beta \simeq 1.010^{-4}\right)$ produces a modulation in the observed CMB dipole with an expected amplitude of $269.1 \mu \mathrm{K}$. We have simulated, accordingly, the reconstruction of the dipole amplitude during one year mission. In Fig. 2 we show the simulated modulation at $90 \mathrm{GHz}$, after subtraction of the Galactic emission assuming $\rho<0.01$. The "observed" signal modulation is then fitted to the expected periodic curve which, to very good 

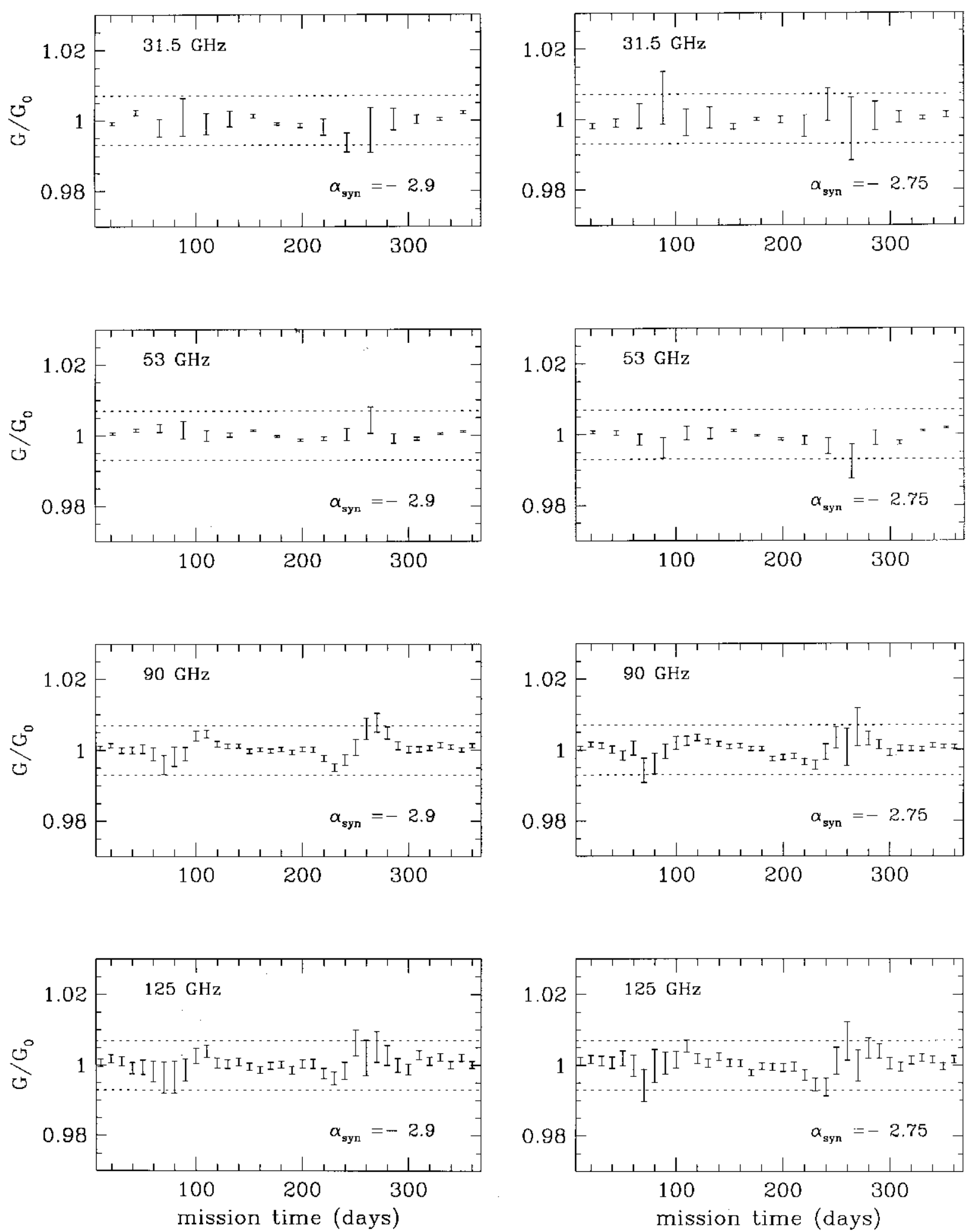

Fig. 1. Absolute calibration against the CMB dipole at the four LFI frequencies. Each point represents the ratio between the reconstructed value, $G$, and the assumed input value, $G_{0}$, of the calibration constant. The results are shown for two assumptions of the synchrotron spectral index: $\alpha_{\mathrm{syn}}=-2.9$ (left hand panels) and $\alpha_{\mathrm{syn}}=-2.75$ (right hand panels). The horizontal limits show the a-priori uncertainty of the CMB dipole amplitude from the COBE-DMR data 


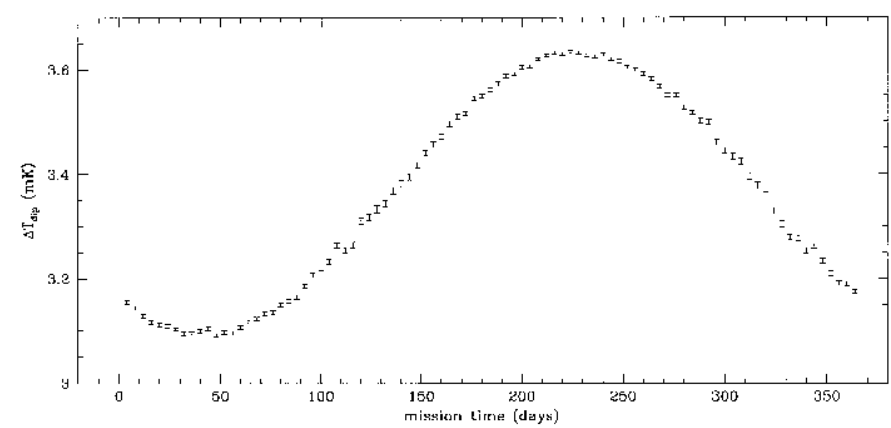

Fig. 2. Simulation of the observed modulation in the value of the CMB dipole due to the orbital velocity of the satellite. This modulation can be used for overall calibration of the maps with accuracy $<0.2 \%$

approximation, is a cosine. The recovered amplitude is $269.2 \pm 0.4 \mu \mathrm{K}$. This result indicates that an overall calibration accuracy of $0.15 \%$ at $90 \mathrm{GHz}$ can be achieved with this method (somewhat degraded accuracies are expected at lower frequencies, due to second order effects from the stronger Galactic emission). This will represent the most accurate overall calibration of the maps on very long time scales ( $\sim 1$ year), and it will be complementary to the shorter term CMB dipole calibration discussed in the previous section.

\subsection{Absolute calibration using external planets}

External planets are good candidates as calibration sources since they provide a signal with suitable intensity and high stability (after correction for slowly varying seasonal effects). Each planet will fall in the COBRAS/SAMBA field of view only about twice per year. Dedicated re-pointing of the satellite is not currently included in the mission plan since it would significantly complicate the observing strategy and increase the required consumables. However, even if occurring occasionally, observations of the external planets will yield a valuable cross check of the CMB Dipole calibration.

The measured antenna temperature of a planet, $T_{\mathrm{A} \text {,planet }}$ can be written as:

$T_{\text {A,planet }}(\hat{\gamma})=\frac{\int T_{\text {planet }}\left(\hat{\gamma}_{\mathrm{p}}\right) J_{n}\left(\hat{\gamma}-\hat{\gamma}_{\mathrm{p}}\right) \mathrm{d} \Omega}{\int J_{n}\left(\hat{\gamma}-\hat{\gamma}_{\mathrm{p}}\right) \mathrm{d} \Omega}$

where $T_{\text {planet }}$ is the frequency-dependent brightness temperature of the planet, $J_{n}\left(\hat{\gamma}-\hat{\gamma}_{\mathrm{p}}\right)$ is the antenna beam pattern, and $\hat{\gamma}$ and $\hat{\gamma}_{\mathrm{p}}$ are the line of sight and planet directions, respectively. Since the planet's solid angle is small compared to the beam size, $\Omega_{\text {planet }} \ll \Omega_{\text {beam }}$, we can approximate Eq. (14) as follows:

$T_{\text {A,planet }}(\hat{\gamma})=T_{\text {planet }}\left(\hat{\gamma}_{\mathrm{p}}\right) \times \frac{\Omega_{\text {planet }}}{\Omega_{\text {beam }}} \times J\left(\left|\hat{\gamma}-\hat{\gamma}_{\mathrm{p}}\right|\right)$,

where $J$ is now assumed to be symmetric.
Assuming the spacecraft at the Lagrangian point L2 in anti-sun configuration, the distance at which the planets will be when in the field of view of the LFI is given approximately by:

$d_{\mathrm{P}} \simeq \frac{\sin (\alpha-\delta)}{\sin (\pi-\alpha)} R_{0}$

where $\delta \simeq \sin ^{-1}\left[A / R_{0} \sin (\pi-\alpha)\right], R_{0}$ is the average planet-Sun distance, and $A \simeq 151.510^{6} \mathrm{~km} \simeq 1.01 \mathrm{AU}$ is the spacecraft-Sun distance (see Fig. 3). The accurate spacecraft-planet distances and relative angular sizes, calculated from the Lissajous orbit around L2 including orbit eccentricity and inclination on the ecliptic, are given in Table 1 for all the pointing events in the first two-years mission. All the listed calibrations will have the planets within $2^{\prime}$ of the beam center. In Table 1 the reference beam is assumed at the center of the array. The entire array will be calibrated within a few days from the indicated time, as the spacecraft spin axis is moved.

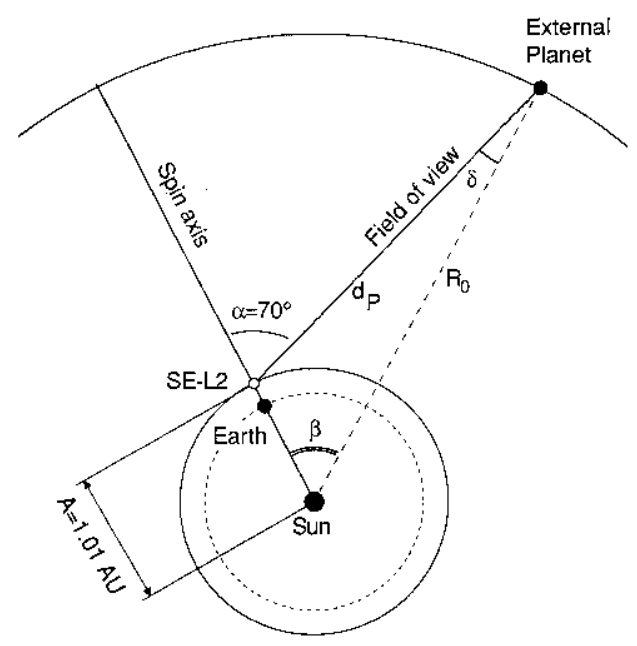

Fig. 3. Spacecraft-Planet geometry when an external planet, $P$, is in the field of view of the COBRAS/SAMBA detectors

Several authors reported measurements of the planets brightness temperature at millimeter wavelengths (e.g. Ulich et al. 1980; Sholherb et al. 1980; Epstein et al. 1980; Ulich 1981; De Pater \& Matisse 1985; Dowling et al. 1987; Rudy et al. 1987; De Pater 1989, 1990; Muhleman et al. 1991). The accuracy of planetary absolute calibration is limited by the uncertainty on the intrinsic radiometric temperatures $T_{\text {planet }}$. We estimate accuracies of $\sim 3 \%$ on $T_{\text {Mars }}, \sim 5 \%$ on $T_{\text {Jupiter }}$ and $T_{\text {Saturn }}$, and $\sim 15 \%$ on $T_{\text {Uranus }}$ and $T_{\text {Neptune }}$. Based on published data and on the above determination of the planets solid angles we derived estimates of the antenna temperatures during calibration as detected by the LFI channels (last four columns of Table 1). 
Table 1. Calibration signals from external planets

\begin{tabular}{|c|c|c|c|c|c|c|c|c|}
\hline$\overline{\text { Planet }}$ & $\operatorname{Day}^{(a)}$ & $\begin{array}{c}d_{\mathrm{P}} \\
(\mathrm{AU})\end{array}$ & $\begin{array}{c}\theta_{\mathrm{P}} \\
(\operatorname{arc}-\mathrm{sec}) \\
\end{array}$ & $\begin{array}{c}\Omega_{\mathrm{P}} \\
\left(10^{-6} \mathrm{deg}^{2}\right)\end{array}$ & $\begin{array}{c}T_{31} \\
(\mathrm{mK})\end{array}$ & $\begin{array}{c}T_{53} \\
(\mathrm{mK}) \\
\end{array}$ & $\begin{array}{c}T_{90} \\
(\mathrm{mK})\end{array}$ & $\begin{array}{l}T_{125} \\
(\mathrm{mK}) \\
\end{array}$ \\
\hline \multirow[t]{2}{*}{ Mars } & 482.83 & 0.664 & 14.06 & 11.97 & 11.83 & 33.87 & 80.03 & 78.50 \\
\hline & 619.66 & 0.896 & 10.42 & 6.575 & 6.49 & 18.60 & 43.95 & 43.11 \\
\hline \multirow[t]{4}{*}{ Jupiter } & 4.25 & 4.994 & 39.32 & 93.69 & 72.53 & 218.7 & 521.9 & 521.9 \\
\hline & 268.58 & 5.034 & 39.01 & 92.21 & 71.38 & 215.2 & 513.7 & 513.7 \\
\hline & 400.58 & 5.020 & 39.12 & 92.73 & 71.78 & 216.4 & 516.5 & 516.5 \\
\hline & 663.66 & 5.008 & 39.21 & 93.17 & 72.13 & 217.5 & 519.0 & 519.0 \\
\hline \multirow[t]{4}{*}{ Saturn } & 188.58 & 8.662 & 19.19 & 22.32 & 15.12 & 44.21 & 106.6 & 106.6 \\
\hline & 319.91 & 8.672 & 19.17 & 22.27 & 15.09 & 44.11 & 106.3 & 106.3 \\
\hline & 567.58 & 8.712 & 19.08 & 22.07 & 14.95 & 43.71 & 105.4 & 105.4 \\
\hline & 699.41 & 8.728 & 19.05 & 21.99 & 14.89 & 43.55 & 105.0 & 105.0 \\
\hline \multirow[t]{4}{*}{ Uranus } & 43.66 & 19.67 & 3.62 & 0.7944 & 0.445 & 1.349 & 3.388 & 3.388 \\
\hline & 184.08 & 19.69 & 3.62 & 0.7930 & 0.444 & 1.346 & 3.382 & 3.382 \\
\hline & 413.00 & 19.69 & 3.62 & 0.7928 & 0.444 & 1.346 & 3.382 & 3.382 \\
\hline & 553.25 & 19.71 & 3.61 & 0.7916 & 0.443 & 1.344 & 3.376 & 3.376 \\
\hline \multirow[t]{4}{*}{ Neptune } & 21.41 & 29.71 & 2.29 & 0.3182 & 0.162 & 0.495 & 1.286 & 1.286 \\
\hline & 163.75 & 29.71 & 2.29 & 0.3181 & 0.162 & 0.495 & 1.286 & 1.286 \\
\hline & 388.91 & 29.70 & 2.29 & 0.3184 & 0.162 & 0.496 & 1.287 & 1.287 \\
\hline & 531.25 & 29.70 & 2.29 & 0.3183 & 0.162 & 0.495 & 1.287 & 1.287 \\
\hline
\end{tabular}

(a) Days are counted from the time of injection of the spacecraft into the Lissajous orbit, after the transfer phase.

The results show that Mars, Jupiter and Saturn have useful signal for calibration. The higher angular resolution at the higher frequencies makes the planets signal at detection much stronger at 90 and $125 \mathrm{GHz}$. However, even at the level of the largest signals $\left(T_{\text {Jupiter }} \simeq 0.5 \mathrm{~K}\right.$ in the higher resolution channels) no significant saturation effects are expected in the LFI receivers. Uranus and Neptune will also be detectable sources with low (few $\mathrm{mK}$ ) signal level, while Pluto will give a $<3 \mu \mathrm{K}$ signal. In Fig. 4 we report the accuracy achieved for absolute calibration using Mars, Jupiter and Saturn for the 31.5, 53 and 90 $\mathrm{GHz}$ receivers, as a 2-hours set of observations (120 spins) takes place. Accuracies of $3 \%$ to $5 \%$ (i.e. the limit imposed by the assumed intrinsic uncertainty in $\left.T_{\text {planet }}\right)$ are generally obtained in the 120 minutes time-scale. In the case of Jupiter the large signal allows to reach such limit in less than 5 minutes.

The CMB dipole is an extended source filling completely the antenna beam, so that the calibration is not sensitive to the details of the beam pattern. On the other hand, when calibrating with sources with small angular size compared to the instrument resolution, such as planets, it is of primary importance to know the beam shape and to take into account pointing errors. The main lobes of the LFI detectors will be measured on ground as part of the testing procedures. In addition, in-flight measurements of the main lobes will be done exploiting the plantes emission itself, as the field of view crosses their positions in the sky. We have simulated the main lobe reconstruction process assuming a Gaussian beam for the $90 \mathrm{GHz}$ channel, and using Jupiter's signal as reference. The results, shown in Fig. 5, demonstrate that the main lobe can be accurately recovered down to about $-20 \mathrm{~dB}$ (see also Delabrouille et al. 1996).

The values of Table 1 are calculated assuming that the planets will fall exactly in the beam center for each scan in the 120 minutes set of observations. Pointing errors can be estimated from the a-posteriori spacecraft pointing stability, which is specified to be $2.5^{\prime}$. Assuming Gaussian beams, this will introduce additional errors of $2.2 \% 0.9 \%$ and $0.4 \%$ for beam size of $12^{\prime}, 18^{\prime}$ and $30^{\prime}$, respectively. The spacecraft will be re-pointed every 120 minutes by $5^{\prime}$. If the planet is in the beam center at a given time, after re-pointing the planet's signal will be attenuated by up to $\sim 10 \%$ (for the $12^{\prime}$ beams). In principle, one can integrate the signal from the planets (or from other point sources) for a time interval longer than 120 minutes by taking into account the change in the beam response due to the re-pointing of the system by using their measured beam shapes. 


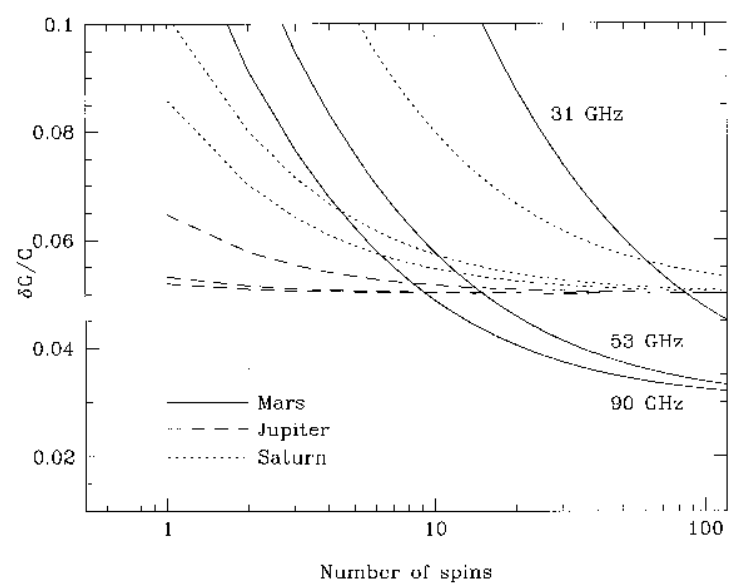

Fig. 4. Absolute calibration accuracies using external planets. The plot shows the convergence to the assumed intrinsic uncertainties $(3 \%, 5 \%$, and $5 \%$ for Mars Jupiter and Saturn respectively) during a 2-hours set of observations (120 spins). For each planet, the upper, middle and lower curves refer to the $31.5 \mathrm{GHz}, 53 \mathrm{GHz}$, and $90 \mathrm{GHz}$ channels, respectively. At $125 \mathrm{GHz}$ the results are very close to those at $90 \mathrm{GHz}$

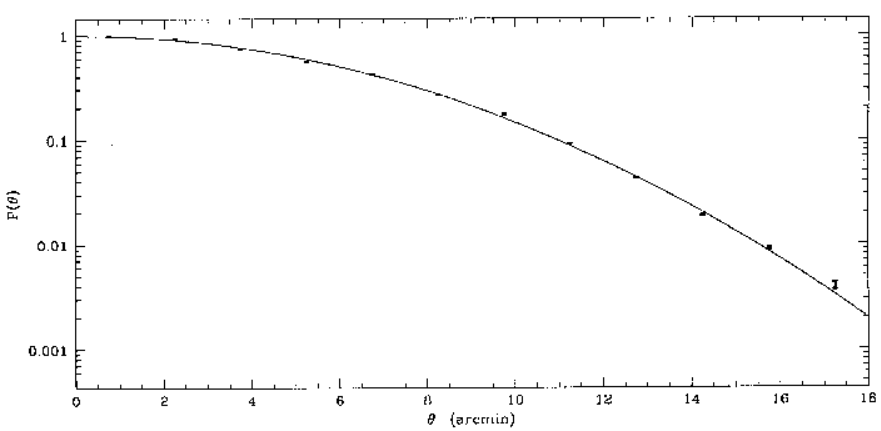

Fig. 5. Reconstruction of the $12^{\prime}$ main lobe of the $90 \mathrm{GHz}$ system using Jupiter as a source.

\section{Relative calibration}

In Sect. 2 we have shown that, in the absence of an accurate a-priori knowledge of the Galactic emission, absolute calibration can be done on time scales of several days. It is important then to address the question of the relative calibration, i.e., to estimate at what level one can control the radiometer gain stability over a time scale of hours or days.

For relative calibration, the accurate knowledge of the observed signals is not critical, as long as it is highly stable within the time scale of interest. By repeatedly observing a stable signal difference $\Delta T$ one can monitor long-term gain changes. If a gain drift $\delta G$ in a time scale $\delta t=t_{2}-t_{1}$ is occurring, the instrument will measure at time $t_{1}$ a signal difference $\Delta V_{1}=\Delta T / G$, and at time $t_{2}$ a signal difference $\Delta V_{2}=\Delta T /(G+\delta G)$. Assuming a well balanced differen- tial system, the measured change in the signal difference is related to the gain change by

$\delta(\Delta V)=\Delta V_{1}-\Delta V_{2}=\frac{\delta G}{G(G+\delta G)} \Delta T \simeq \frac{\delta G}{G^{2}} \Delta T$.

From Eq. (5), the accuracy with which one can account for variations in the gain is:

$$
\left(\frac{\sigma_{\delta G}}{G}\right)_{\mathrm{rel}}=\frac{G}{\Delta T} \sigma_{\Delta V}=\sqrt{2} \frac{\delta T_{\mathrm{rms}}}{\Delta T}
$$

where $\delta T_{\text {rms }}$ is the rms noise associated with the measurement of $\Delta T$, according to Eq. (6). So we obtain:

$$
\left(\frac{\sigma_{\delta G}}{G}\right)_{\mathrm{rel}}=\frac{\sqrt{2} K T_{\mathrm{sys}}}{\Delta T \sqrt{\Delta \nu \tau}}
$$

Equation (8), as expected, reduces to Eq. (20) when $\sigma_{\Delta T}=0$, since in this case the accuracy of the calibration load temperatures is not relevant.

The time scale of the expected gain drifts depends on the source of instability. Gain variations can be due to thermal changes or can be intrinsic of the detectors. The differential receiver minimizes the effect of intrinsic $1 / f$ noise. In addition, residual effects on time scales $<2$ hours are averaged out by the large number (typically $\approx 1000$ ) of successive measurements of the same sky pixel (observed by different radiometers at a given frequency), with the net effect of a small increase of the nominal white noise level of the receivers (e.g. Janssen et al. 1996). Our main concern here is then our ability of tracing slow drifts due to residual $1 / f$ noise on time-scales $>2$ hours. Laboratory measurements suggest that one may expect gain variations of order $\delta G / G \approx 0.5 \%$ in few hours, or $\delta G / G \approx 1 \%$ in $\sim 24$ hours (Weinreb 1996).

Possible thermal effects on the gain are quite small, thanks to the choice of the L2 orbit and scanning technique. Thermal variations can only arise due to re-pointing maneuvers away from the anti-sun direction. A thermal study (Bersanelli et al. 1996) shows that any temperature drifts at the focal plane will be dominated by a slowly varying component with a time-scale of $\sim 100$ hours, so that for time scales $\ll 100$ hours thermal effects can be considered linear. Thermal effects on the gain are expected only at a level $\delta G / G=0.1 \%$ in the 100 hours period.

We have simulated the presence of a linear drift in the noise-added data stream with a rate of $\delta G / G \simeq 0.25 \%$ per hour. As shown in Sect. 1, in order to disentangle a gain drift from a baseline drift in the internal load (which will be discussed in the next section), we need to monitor signal differences rather than the signal intensity in a fixed pixel. We follow the circle described by the beam in two hours. The voltage associated to each pixel is

$V_{i}\left(t_{k}\right)=\frac{T_{i}\left(t_{k}\right)}{G[1+(\delta G / G) t]}$ 
where $G$ is the gain at an arbitrary time $t=0, t_{k+1}-$ $t_{k}=1 \mathrm{~min}, k=1,2, \ldots 120$, and $T_{i}\left(t_{k}\right)=T_{\mathrm{Gal}, i}+T_{\mathrm{Dip}, i}-$ $T_{\text {eff }}\left(t_{k}\right)$ is the temperature relative to the internal load of the $i$-th pixel at time $t_{k}$ due to the Galaxy and the CMB dipole. We then evaluate $\Delta T_{i}\left(t_{k}\right)$, the difference between $T_{i}\left(t_{k}\right)$ and the signal of that pixel in the circle where the dipole is expected to provide the minimum contribution. We assume that the instrument response is stable during each spin period ( 1 minute). Since $\delta G / G \ll 1$, we can write:

$\Delta V_{i}\left(t_{k}\right) \simeq \frac{\Delta T_{i}\left(t_{k}\right)}{G}-\frac{\Delta T_{i}\left(t_{k}\right)}{G} \frac{\delta G}{G} t$.

Note that the load temperature (and its possible drift occurring on time scales $\gg 1 \mathrm{~min}$ ) has been eliminated. Now we can average the previous expression over a circle obtaining

$\left\langle\Delta V_{i}\left(t_{k}\right)\right\rangle=\left\langle\frac{\Delta T_{i}\left(t_{k}\right)}{G}\right\rangle-\left\langle\frac{\Delta T_{i}\left(t_{k}\right)}{G}\right\rangle \frac{\delta G}{G} t$

where the notation $\langle\ldots\rangle \equiv N^{-1} \sum_{i=1}^{N}, N$ being the number of elements in a given circle. Note that in this case the Galactic emission provides additional modulation to the signal observed in each circle, thus contributing to the detection of gain drifts. We have then a set $\left\langle\Delta V_{i}\left(t_{k}\right)\right\rangle$ (and associated noise) with $k=1,120$, that we can fit to a linear law $y=a+b t$. The ratio between the angular coefficient $b$ and the zero-value $a$ provides the estimate of the gain drift. Due to the slow gradient of the dipole, the time scale can be expanded up to $\sim 10$ successive circles with basically no additional uncertainty on the result. This allows to further averaging the values of $\left\langle\Delta V_{i}\left(t_{k}\right)\right\rangle$ already evaluated for each circle.

The results of our calculations are presented in Fig. 6 for the four LFI frequencies. Each point in the plot represents the algorithm reconstruction of a drift of $0.25 \%$ per hour (or $0.5 \%$ per circle) based on 6 consecutive circles (corresponding to 12 hours of data). Again, the accuracy of the results is degraded significantly in the two periods of the year when $\left\langle\Delta V_{i}\left(t_{k}\right)\right\rangle$ is smaller due to the geometry of the CMB dipole, which dominates in general over the Galaxy. On average, however, it is apparent that the relative calibration allows to recover the gain drift with high accuracy at all frequencies, with a typical uncertainty of less than $1 \%$ on $G$ for the assumed 12 hours time scale. Our simulations show that this result is nearly independent of the amplitude of the assumed drift.

This demonstrates that even in the absence of frequent absolute calibration, relative calibration can provide an accurate monitor of the long-term gain stability.

\section{Removal of thermal baseline drifts}

Besides gain changes, another potential effect of temperature drifts in the focal plane will be a linear drift $\delta T_{\text {eff }}$ in the reference load temperature, appearing in the signal as a baseline change with typical time scale $\sim 100$ hours. We have simulated the presence of a baseline drift $\delta T_{\text {eff }}=40 \mu \mathrm{K}$ per hour, a conservative estimate based on the current thermal study of the mission, to demonstrate the ability to accurately remove their potential effects.

We simulate the observations in one year in nominal configuration. As the spin axis moves every two hours by $5^{\prime}$, in the thermal time-scale ( $\sim 100$ hours) the beam observes $\sim 50$ different circles, with some fraction of overlap among them. The signal measured in each elements of the circle is

$G \times V_{j}^{\text {obs }} \equiv T_{j}^{\text {obs }}=\left(\Delta T_{j}^{\text {true }}+\sigma_{j}\right)+\delta T_{\text {eff }} t(h)$

where $\Delta T_{j}^{\text {true }} \equiv T_{n}^{\text {true }}-T_{\text {ref }, n}$ is the difference between the true sky signal and the reference at an arbitrary time $t=0$, and $\sigma_{j}$ is the statistical noise of the receiver. We assume that the elements of 3 adjacent circles observed the same signal on the sky, a reasonable approximation because of the slow gradients of the dipole and of the Galactic emission. So we average Eq. (23) over the 3 circles obtaining

$\left\langle T_{j}^{\text {obs }}\right\rangle=\Delta T_{j}^{\text {true }}+\left\langle\sigma_{j}\right\rangle+\delta T_{\text {eff }} t(h)$.

Now we fit this average with a linear law

$\left\langle T_{j}^{\mathrm{obs}}\right\rangle=a_{j}+b_{j} t$.

Thus, $a_{j}$ provides the estimate of $\Delta T_{j}^{\text {true }}+\left\langle\sigma_{j}\right\rangle$, while $b_{j}$ is the estimate of the thermal drift. The accuracy of the estimate improves by a factor 4 by using the $\sim 16$ estimates of $b_{j}$ to reach the 100 hours baseline (corresponding to $\sim 50$ circles).

Note that while absolute and relative calibration use the observed signal modulation, here the result is independent of the observed signals, and it depends on the LFI frequency channels only because of their different intrinsic noise and angular resolution. The accuracy in reconstructing the thermal drift is determined by the signal to noise ratio in each pixel. From our simulations we conclude that we can recover the correct value of the thermal drift with an accuracy of $\lesssim 1 \%$ (at the 1 sigma level). This introduces errors which are $\lesssim 1 \mu \mathrm{K}$ on the estimate of the CMB anisotropy amplitude.

The most potentially dangerous thermal effects are those with a component synchronous with the spin rotation. In fact, in this case any spurious signal variation will be integrated over long time periods and will easily exceed the final integrated statistical noise. An accurate thermal study shows that for the COBRAS/SAMBA payload in L2 Lissajous orbit and in total-shadow condition the only spin-synchronous effect can come from small asymmetries of the solar panel plane with respect to the spin axis. Even in the most critical configuration (which happens to be when pointing $15^{\circ}$ away from anti-sun direction) 

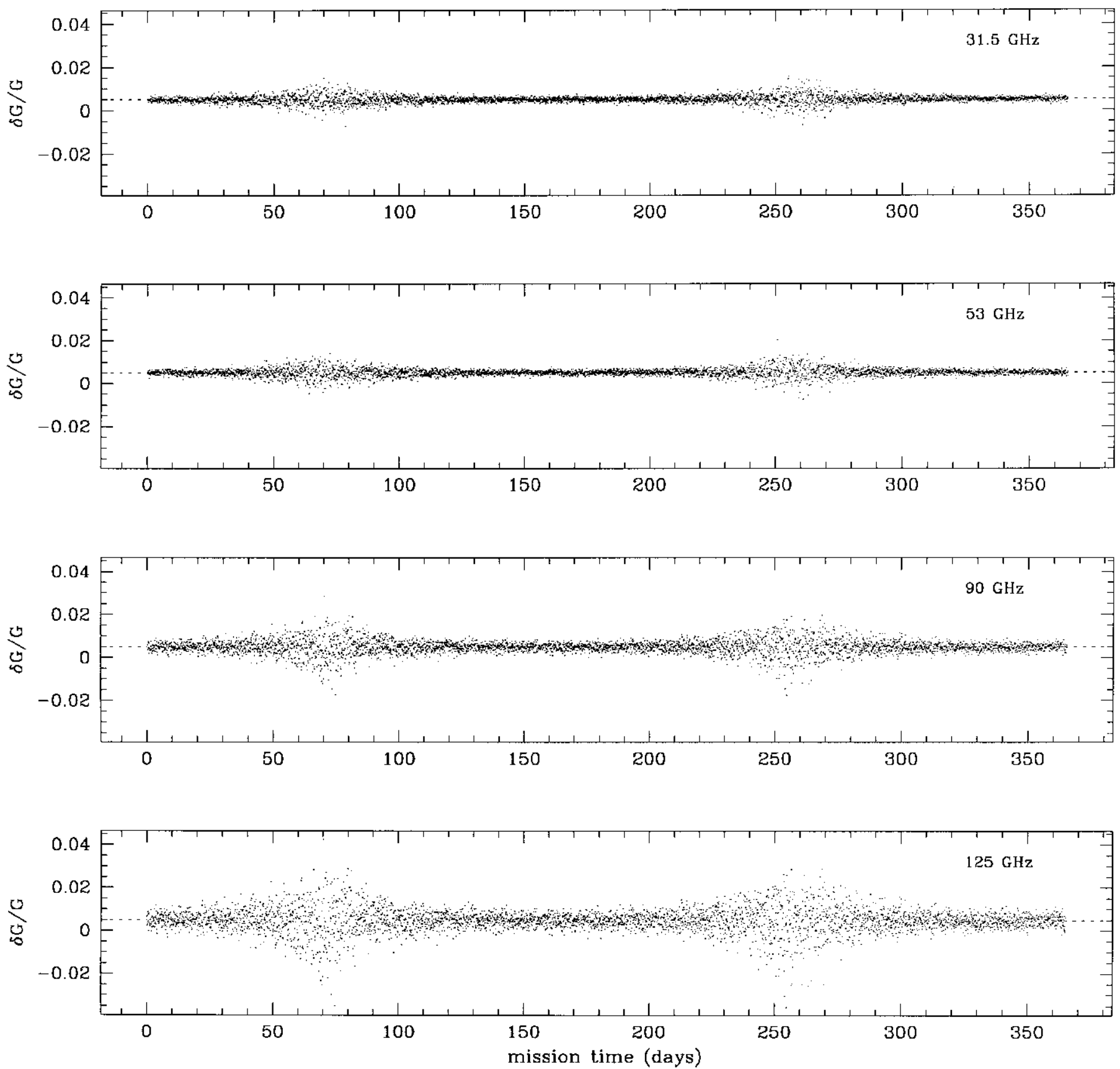

Fig. 6. Reconstruction of long-term gain drifts (relative calibration) from the closure pattern of the scanning technique. A drift $\delta G / G=0.0025 /$ hour was added to the data. Here the results are presented for the drift as reconstructed in each 2-hour circle, corresponding to $\delta G / G=0.005$ (dashed horizontal lines). Each point represents the simulation results based on 6 hours of observation. The regions of increased spread correspond to the periods when the scan geometry is such to have minimum sky signal modulation within the observed circle 
the synchronous component of the physical temperature modulation is expected to be less than $1 \mu \mathrm{K}$, and any effects on the signals observed by the COBRAS/SAMBA instruments can be considered negligible. This is a major advantage of the choice of the Sun-Earth L2 orbit, which provides the best possible thermal environment for the mission.

\section{Conclusions}

The COBE-DMR experiment was calibrated using the small dipole seasonal component due to the Earth's revolution and the signal observed from the Moon. The absolute calibration was fitted over the entire mission lifetime and checked on the short time scale using stable noise sources (Bennett et al. 1992). In this work we have shown that a second generation space mission for high-resolution imaging of the $\mathrm{CMB}$ can rely for calibration on the COBE measurement of the CMB dipole, making the non-trivial implementation of an active calibration system not necessary. We find that even with a very conservative approach to remove the effects of Galactic contamination, the COBRAS/SAMBA Low-Frequency Instrument can be calibrated with $<0.7 \%$ accuracy (i.e. limited by the DMR measurement of the dipole) with a frequency of 10 to 20 days. In reality, more efficient removal of the Galactic emission will be possible due to the multifrequency nature of the experiment, leading to more frequent and precise calibration.

Measuring the calibration over 1 year of observations, would lead to extremely small statistical errors $(<0.1 \%)$ even with strict values for the threshold $\rho$, but the ultimate accuracy will be limited by the $\sim 0.7 \%$ a-priori uncertainty. This uncertainty does not affect the precision of the dipole subtraction procedure and leads to an uncertainty of a fraction of $\mu \mathrm{K}$ in the estimate of the primary anisotropy. This is adequate for the scientific goals of the proposed COBRAS/SAMBA mission. However, an even greater accuracy can be achieved for the long-term calibration by exploiting the spacecraft orbital motion. The seasonal proper velocity is known a-priori with extreme accuracy, allowing absolute calibration of the maps to better than $0.2 \%$.

The observation of external planets will provide, a few times per year, independent absolute calibration with 3 to 5 percent accuracy. This will be a useful independent cross-check of the primary absolute calibration based on the CMB dipole anisotropy. The accuracy of planetary calibration is subject to improvement if progress is made in measurements and understanding of the planets' microwave emission. In addition, the signal from the planets can be used to accurately map the shape of the system main lobes.

Thermal variations or intrinsic instrumental effects can perturb the stability of the gain and of the instrument baseline. Short time-scale effects are efficiently diluted by the redundant observation of the same sky pixels for several scans as seen from different detectors. In this work we have concentrated on analyzing drift effects over several hours time scale, which may cause striping and systematic errors in the maps. Our simulations show that the stability of the calibration constant can be monitored with $\lesssim 1 \%$ accuracy over time scales of $\sim 6$ hours and that thermal drifts of the instrument baseline (occurring typically on $\sim 5$ days time scale) can be controlled to within $1 \%$. These results ensure that instrumental systematic effects, if significant, can be readily recognized from the data, and that no artifacts will appear in the final maps.

The accuracy with which one can remove such effects from the data depends strongly on the instrument sensitivity as well as on the observing strategy. In addition, a very stable thermal environment and a large thermal constant at the focal plane instrument greatly simplify the analysis of thermal effects, by making them linear over all the time scales of interest. In the case of COBRAS/SAMBA, these conditions are achieved by a proper choice of the orbit (Sun-Earth L2 provides the best possible thermal conditions) and a careful design of the thermal architecture of the payload.

\section{References}

Bennett, et al., 1992, ApJ 391, 466

Bersanelli M., et al., 1995, ITESRE Rep. 177/95

Bersanelli M., et al., 1996, COBRAS/SAMBA Report on the Phase A Study, ESA D/SCI(96)3

Bouchet F.R., Gispert R., Puget J.L., 1995, astro-ph/9507032

Cheng E., Cottingham D.A., Fixen D.J., et al., 1996, ApJ 456, L71

Clapp, et al., 1994, ApJ 433, L57

Danese L., et al., 1996, Astroph. Lett. Comm. 33, 257

De Bernardis P., De Gasperis G., Masi S., Vittorio N., 1994, ApJ 433, L1

Delabrouille J., et al., 1996 (in preparation)

De Pater I., Matisse S.T., 1985, Icarus 62, 143

De Pater I., et al., 1989, Icarus 79, 190

De Pater I., 1990, ARA\&A 28, 347

Dowling T.E., Muhleman D.O., Berge G.L., 1987, Icarus 70, 506

Epstein, et al., 1980, Icarus 41, 103

Gundersen J.O., et al., 1995, ApJL 443, L57

Janssen M.A., Lawrence C.R., 1995, Astroph. Lett Comm. 32, 289

Janssen M.A., et al., 1996, ApJ, astro-ph/9602009 (submitted)

Jungman G., Kamionkowski M., Kosowski A., Spergel D.N., astro-ph/9512139

Haslam C.G.T., et al., 1982, A\&AS 47, 1

Kogut A., et al., 1993, ApJ 419, 1

Kogut A., et al., 1996, ApJ (in press)

Lawson D.K., et al., 1987, MNRAS 225, 307

Lineweaver C., et al., 1996, ApJ (submitted)

Mandolesi N., et al., 1995, Astroph. Lett Comm. 32, 309

Mandolesi N., et al., 1996 (in preparation)

Mather J.C., et al., 1994, ApJ 420, 439 
Muciaccia F., et al., 1996, A\&A (in press)

Muhleman D.O., Berge G.L., 1991, Icarus 92, 263

Netterfield C.B., et al., 1995, ApJ 445, L69

Rudy D.J., et al., 1987, Icarus 71, 159

Ruhl J.E., Dragovan M., Platt S.R., Kovac J., Novak G., 1995, ApJL 453, 1

Smoot G.F., et al., 1990, ApJ 360, 685

Smoot G.F., et al., 1992, ApJ 396, L1
Sholherb F.P, et al., 1980, Icarus 42, 125

Ulich B.L., et al., 1980, IEEE Trans. Antennas Propagat., AP-28, 367

Ulich B.L., 1981, AJ 86, 1619

Wright E.L., Hinshaw G., Bennett C.L., 1996, ApJL, astro-ph/9510102 (submitted)

Weinreb S., 1996 (private commumication)

Witebsky C., 1988 (private communication) 